\title{
A Reliable Method for Fabricating sub-10 nm Gap Junctions Without Using Electron Beam Lithography
}

\author{
Y. Naitoh \\ Motorola Labs, Corporate RED, Motorola Japan Ltd., \\ 3-20-1, Minami-azabu, Minato-ku, Tokyo 106-8573, Japan \\ and Nanotechnology Research Institute and Research Consortium for Synthetic Nano-Function Materials Project, \\ National Institute of Advanced Industrial Science and Technology 1-1-1, Higashi, Tsukuba, Ibaraki 305-8562, Japan
}

\section{K. Tsukagoshi}

Japan Science and Technology Corporation 4-1-8, Honcho, Kawaguchi, Saitama 332-0012 Japan and Nanotechnology Research Institute, National Institute of Advanced Industrial Science and Technology 1-1-1, Higashi, Tsukuba, Ibaraki 305-8562, Japan

K. Murata and W. Mizutani*

Nanotechnology Research Institute, National Institute of Advanced Industrial Science and Technology 1-1-1, Higashi, Tsukuba, Ibaraki 305-8562, Japan

(Received 30 May 2003; Accepted 2 June 2003; Published 5 June 2003)

\begin{abstract}
We demonstrate a high yield production scheme to fabricate sub-10 nm co-planar metal-insulator-metal junctions without using electron beam lithography. The fabricating procedure contains two photolithographys followed by shadow evaporation. Ultrasmall gaps were formed in the crossing region of the two metal layers during the evaporation of the second layer. The sizes of the gaps were estimated using scanning electron microscopy images. Poly (3-hexylthiophene-2,5-diyl) layers were deposited on the junctions using a special ink-jet technique. The results of the conductivity measurement of the molecular layer indicate that these junctions can be used in the study of molecular sensors.
\end{abstract}

Keywords: Scanning electron microscopy (SEM); Nanostructure chemistry, processing and fabrication; Nano-electronics and related devices; Gold; Silicon Oxide; Shadow evaporation; Photolithography; Molecular sensor.

\section{INTRODUCTION}

Molecules were proposed for use as active electrical devices as early as 1974, when Aviram and Ratner theoretically demonstrated that uinimolecular rectification, or an asymmetrical electrical property, should be obtained for a single molecule [1]. Because organic molecules are one of the smallest functional materials and can be massproduced as Avogadro constant order with molecular synthesis techniques, molecular devices have attracted attention as minute and low-price devices.

Recently, the miniaturization limits of fabrications are approaching the molecular size. Narrow gap electrodes have found wide acceptance in the study of the electrical properties of molecules [2-3]. Meanwhile, a variety of techniques for narrow gap junction fabrication have already been demonstrated. One of the most popular techniques is electron beam lithography (EBL) [4-9]. Commercial EBL systems have the ability to focus electrons to diameters less than $10 \mathrm{~nm}$, enabling the fabrication of structures at the nanometer level. However, because the EBL is highly cost-and-time-consuming, procedures that do not use EBL are preferred for industrialization. Other techniques applicable to nano-fabrication include electromigration [10], shadow evaporation [11], mechanical break junction [3], and electroplating [12]. In these techniques, the gaps were fabricated without EBL. However, all the above techniques require nano-scale prestructures fabricated by EBL. No fabrication process that re-

*Corresponding author: W.Mizutani@aist.go.jp

quires preparation without using EBL while using only $\mu \mathrm{m}$ order patterning has been reported as far as we know.

In this communication, we demonstrate a high yield production scheme to fabricate sub-10 nm co-planar metal-insulator-metal junctions without using EBL. The fabricating procedure contains two photolithographys followed by shadow evaporation and does not contain any EBL process. Figure 1 shows a schematic representation of the nanogap electrodes. Ultrasmall gaps are formed in the crossing region of the two metal deposited layers during the evaporation of the second layer. The sizes of the ultrasmall gap can be controlled by the height of the first metal layer and the angles of the two evaporations. With this method, we could make metal electrodes with intervals ranging from 5 to $60 \mathrm{~nm}$. The fabricated

Top view

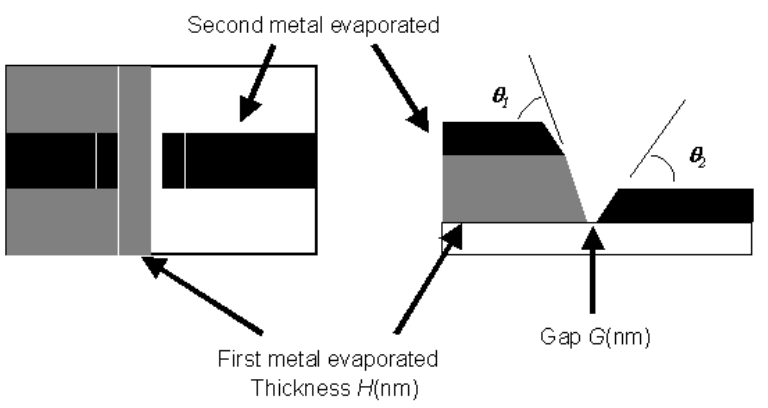

FIG. 1: Side view and top view of two perpendicular metal strips evaporated on a substrate. The step height of the first layer causes a discontinuity in the second layer in the crossing region. 
Top view

(a)

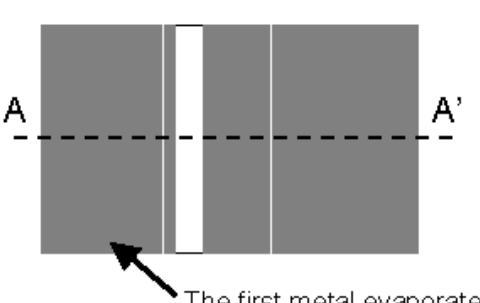

The first metal evaporated

(b)
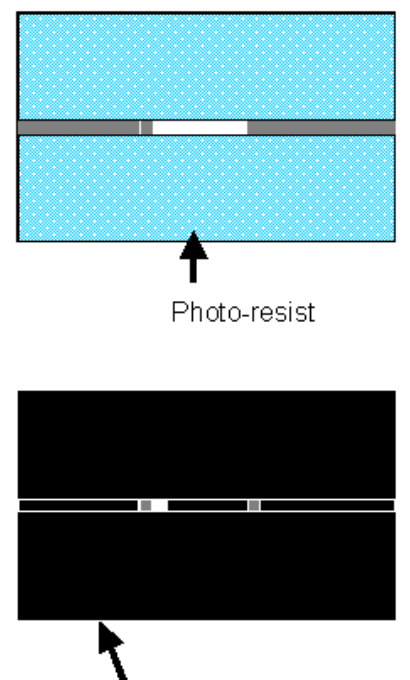

Side view
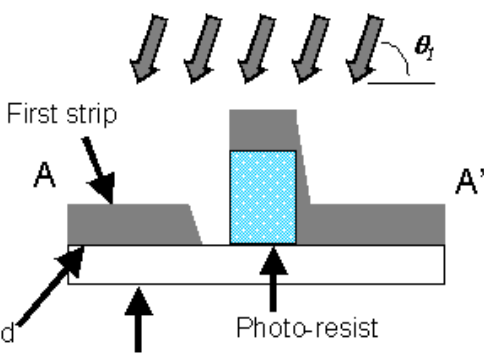

$\mathrm{SiO}_{2}(300 \mathrm{~nm}) / \mathrm{Si}$ substrate

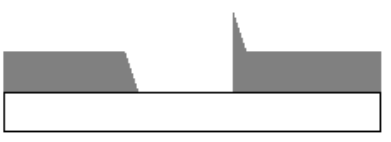

$\boldsymbol{\theta}_{2}$
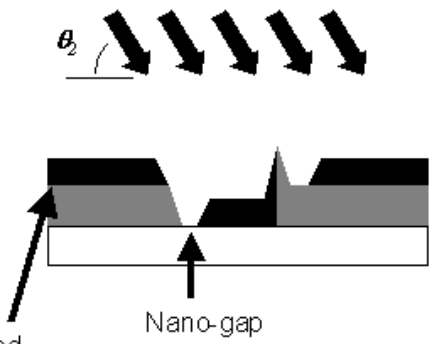

FIG. 2: Schematics show side views and top views for the first evaporation (a), the second patterning (b), and the second evaporation (c). Side views show cross sections along dashed line A-A'.

gaps showed very high resistance of $\sim \mathrm{T} \Omega$. While with molecules connecting the electrodes over the gap, we observed characteristic conductance reflecting the molecular properties. Poly (3-hexylthiophene-2,5-diyl) layers were deposited on the electrodes using a special ink-jet technique. The measured resistance of the molecular layer was clearly changed with the gap sizes. We considered that the change reflects the characteristic behavior of the nano-scale systems. The results indicate that these junctions can be used in the study of molecular devices.

\section{ELECTRODE PROCESSING}

Figure 2 shows a schematic diagram of the fabrication process. A 1000-nm thick uniform film of resist material (AZ5214E, Clariant Japan) was spun on $\mathrm{SiO}_{2}(300 \mathrm{~nm}) / \mathrm{Si}$ wafers. They were patterned by conventional photolithography. After the first patterning, the first metal layer (2 $\mathrm{nm} \mathrm{Cr}$ and 25-60 nm Au) was deposited with an angle $\theta_{1}$ from the substrate surface (Figure 2 (a)). After liftoff, the second lithography process was performed with a mask of slits orthogonal to the slit on the first layer (Figure $2(\mathrm{~b}))$. The width of the second slit is $2 \mu \mathrm{m}$, which is the minimum line width of our photolithography. The second metal layer ( $2 \mathrm{~nm} \mathrm{Cr}$ and $20 \mathrm{~nm} \mathrm{Au}$ ) was deposited with an angle $\theta_{2}$ from the opposite side of the first deposition (Figure 2(c)). Because the height of the first strip prevented the formation of a continuous second strip at $\theta_{1}<\theta_{2}$, a narrow gap was formed between the two strips. These angles of the step edges almost agree with the angle of evaporation. We adopted the condition of both angles $\left(\theta_{1}, \theta_{2}\right)$ to be less than 90 degree. The reason for using $\theta_{1}<90^{\circ}$ for the angle $\theta_{1}$ is that a flat and sharp step wall of the first metal strip is necessary for generating a constant width gap along the $\mu \mathrm{m}$ length. When we evaporate the first layer at $\theta_{1}=90$ degrees, we cannot avoid the part where the evaporation angle exceeds 90 degrees, because evaporation sources have a finite distribution. The evaporation at over 90 degrees produces a protruding part along the sidewalls of the photo-resist. This protrusion prevents the formation of a constant gap width. The gap-size $G$ can be controlled by the thickness of the first layer $H$ and the edge angle of the two strips $\theta_{1}$ and $\theta_{2}$. If we neglect the migration of metal atoms, the gap-width can be estimated as follows:

$$
G=H \cdot\left(\operatorname{cotan} \theta_{2}-\operatorname{cotan} \theta_{1}\right) .
$$

After lift-off, the electrodes were cleaned in oxygen plasma before imaging by scanning electron microscopy (SEM) in order to estimate the small gap width. An estimation of the leak current was then carried out on a probe station. 

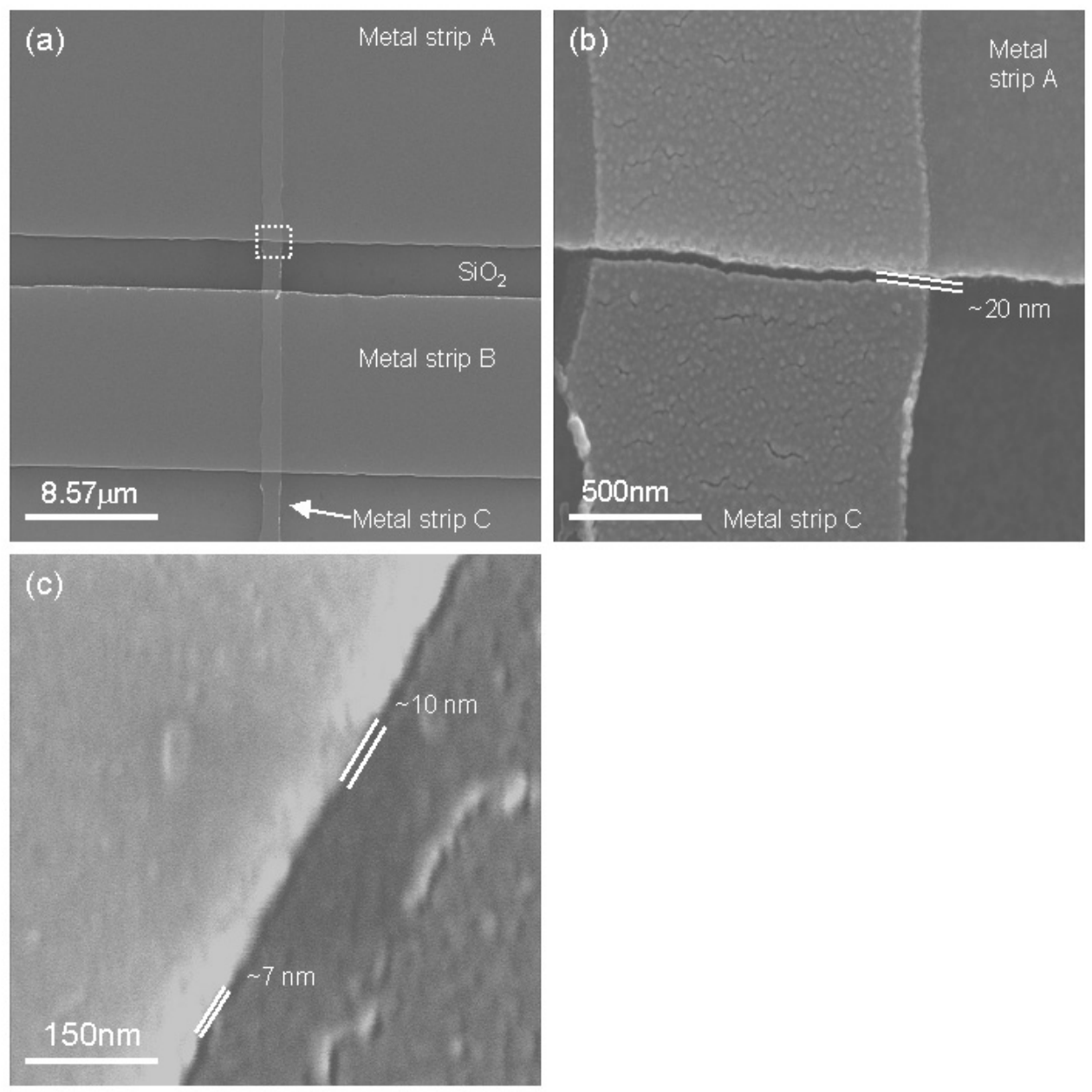

FIG. 3: Characterization of the electrodes. (a) Representative SEM image of the $G=20 \mathrm{~nm}$ electrodes. (b) A magnified image of the area indicated by the white dashed rectangle in (a). (c) Representative SEM image of the $G \sim 10 \mathrm{~nm}$ electrodes.

\section{RESULTS}

Figure 3 (a) shows representative SEM images of the fabricated electrodes of $G=20 \mathrm{~nm}\left(H=50 \mathrm{~nm}, \theta_{1}=60^{\circ}\right.$, and $\left.\theta_{2}=45^{\circ}\right)$. The metal strips $\mathrm{A}$ and $\mathrm{B}$ were deposited during the first evaporation and metal strip $\mathrm{C}$ was deposited during the second evaporation. Figure $3(\mathrm{~b})$ shows a magnified image at the rectangular mark in Figure (a). A small gap was observed at the intersection of metal strips A and C. The yield of the 20-nm gaps is over $90 \%$. Figure 3 (c) shows an image of the 10- $\mathrm{nm}$ gap electrodes $\left(H=40 \mathrm{~nm}, \theta_{1}=75^{\circ}\right.$, and $\left.\theta_{2}=60^{\circ}\right)$. Under this condition, we observed several points showing narrower intervals than the value expected from equation (1) along the edges. We considered that a migration of metal or an uneven edge of the first metal strip caused such narrower intervals. The yields of the $10-\mathrm{nm}$ and 5 -nm gaps $\left(H=25 \mathrm{~nm}, \theta_{1}=75^{\circ}\right.$, and $\left.\theta_{2}=60^{\circ}\right)$ were about $55 \%$ and $20 \%$, respectively. Most of the remaining electrodes showed a short-circuit (under $10^{3} \Omega$ order). Because the short-circuit might be caused by bridging metal atoms between the intervals, the migration and the uneven edges are a serious problem for the fabrication of sub10-nm gap electrodes. However, such bridges are only small parts of the entire gap. The short-circuit points are easily removed using an electromigration method [10]. The final yields of the sub 10-nm gap fabrication are improved to over $80 \%$ by the combination of the electromigration and our procedure. With our fabrication procedure, we made metal electrodes with gaps ranging from 5 to $60 \mathrm{~nm}$ with a high reproduction.

Next, we demonstrated an application of the fabricated electrodes. Poly (3-hexylthiophene-2, 5-diyl) layers were deposited using a special ink-jet technique on three electrodes with $G=10,20$, and $60 \mathrm{~nm}$. Figure 4 (a) shows an SEM image of the molecular layer on the fabricated electrodes. The thickness of the molecular layer was about $150 \mathrm{~nm}$, which was measured using atomic force microscopy. Figure 4 (b) shows the representative $I$ - $V$ characteristics of the molecular layer on the three gap electrodes. The $I$ - $V$ measurements were carried out in air and in the dark at room temperature with the voltage applied between electrode 1 and electrode 2. The molecular layers were supposed to be doped with oxygen. The resistances of the bare gap electrodes were over $10 \mathrm{~T} \Omega$. After the deposition of the molecular layers, the measured resistances were $5 \mathrm{k} \Omega, 280 \mathrm{k} \Omega$, and $200 \mathrm{M} \Omega$ for the $10-\mathrm{nm}$, 20-nm, and 60-nm gap electrodes, respectively. The resistivity of the molecular layer on the 60-nm gap electrodes was on the order of $10^{5} \Omega \mathrm{cm}$, which agreed with our previous measurements using the $10-\mu \mathrm{m}$ gap electrodes. For a bulk material, the resistance is in inverse proportion 

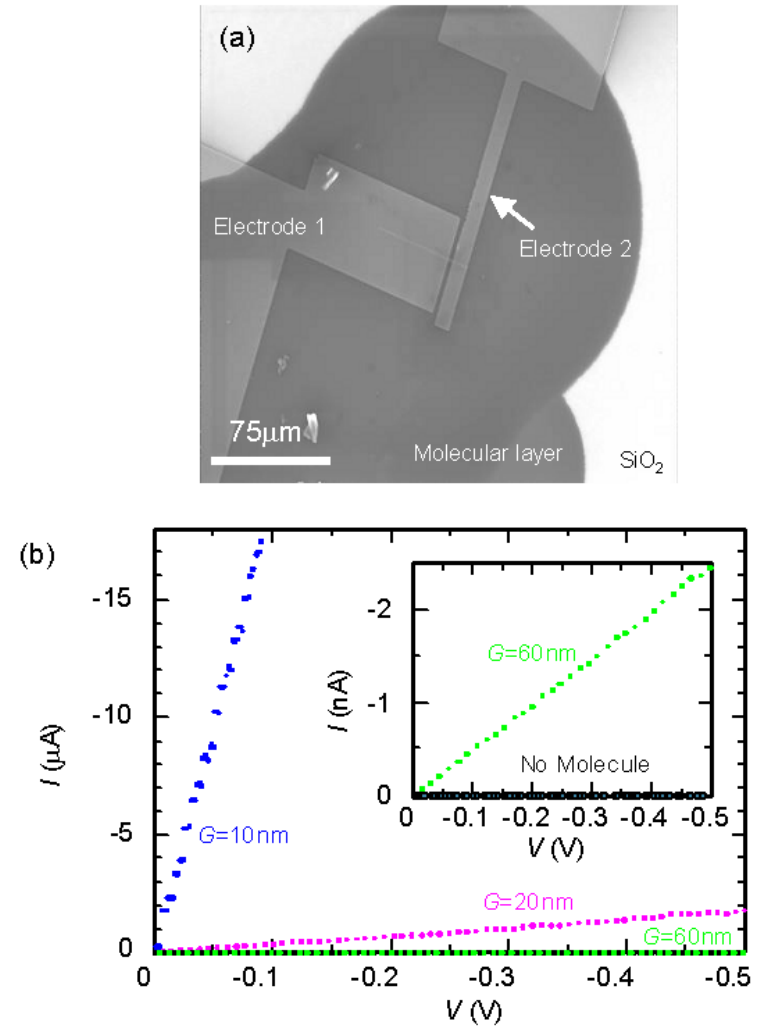

FIG. 4: (a) SEM image of a deposited molecular layer on the fabricated electrodes. (b) $I$ - $V$ characteristic of bare electrodes and molecular layers deposited on $G=10,20$, and $60 \mathrm{~nm}$ electrodes.

to the gap size. However, the resistances of the gaps of nearly molecular length are clearly lower than the value expected from the scale change. We conjectured that the deposited molecular layers were amorphous consisting of small domains of ordered molecules. When the small do- mains directly bridge between gaps, the resistances should become lower than that of the amorphous molecular layer, which may be dominated by the inter-domain resistances. The low resistances observed for the $10-\mathrm{nm}$ and $20-\mathrm{nm}$ gap electrodes might be caused by the direct bridging of the small domains. Such details are now under investigation, but we considered that the result is a characteristic behavior of nano-scale systems.

\section{CONCLUSION}

In conclusion, we have developed a high yield production scheme to fabricate sub-10-nm co-planar metalinsulator-metal junctions. Our fabrication procedure contains two photolithographys followed by shadow evaporation. Electron beam lithography was not needed in our procedure. Ultrasmall gaps were formed in the crossing region of two metal layers during the evaporation of the second layer. The yields of the sub $10-\mathrm{nm}$ gaps are improved to over $80 \%$ by the combination of this procedure and electromigration. Poly (3-hexylthiophene-2,5-diyl) layers were deposited on the junctions using a special inkjet technique. The measured resistances of the molecular layers were lower than the values expected from the bulk property. The results of the conductivity measurements of the molecular layer indicate that these junctions can be used in the study of molecular sensors.

\section{Acknowledgments}

This work was partly supported by NEDO under the Nanotechnology Materials Program and also supported by MEXT under the NanoProcessing Partnership Program.
[1] A. Aviram and M. A. Ratner, Chem. Phys. Lett. 29, 277 (1974).

[2] M. A. Reed, C. Zhou, C. J. Muller, T. P. Burgin, and J. M. Tour, Science 278, 252 (1997).

[3] H. Park, J. Park, A. K. L. Lim, E. H. Anderson, A. P. Alivisatos, and P. L. McEuen, Nature 407, 57 (2000).

[4] P. B. Fischer and S. Y. Chou, Appl. Phys. Lett. 62, 2989 (1993).

[5] S. Itoua, C. Joachim, B. Rousset, and N. Fabre, Nanotechnology 5, 19 (1994).

[6] E. Di Fabrizio, L. Grella, M. Gentili, M. Baciocchi, L. Mastrogiacomo, and M. Morales, Jpn. J. Appl. Phys. 36, (1997) L70.

[7] M. A. Guillorn, D. W. Carr, R. C. Tiberio, E. Green- baum, and M. L. Simpson, J. Vac. Sci. Technol. B 18, 1177 (2000).

[8] K. Liu, Ph. Avouris, J. Bucchignano, R. Martel, S. Sun, and J. Michl, Appl. Phys. Lett. 80, 865 (2002).

[9] M. S. M. Saifullah, T. Ondarcuhu, D. K. Koltsov, C. Joachim, and M. E. Welland, Nanotechnology 13, 659 (2002).

[10] H. Park, A. K. L. Lim, A. P. Alivisatos, J. Park, and P. L. McEuen, Appl. Phys. Lett. 75, 301 (1999).

[11] G. Philipp, T. Weimann, P. Hinze, M. Burghard, and J. Weis, Microelectron. Eng. 46, 157 (1999).

[12] A. F. Morpurgo, C. M. Marcus, and D. B. Robinson, Appl. Phys. Lett. 74, 2084 (1999). 